\title{
Subcutaneous Adipose Tissue Cell-derived Cytokines Are Early Markers of Impaired Glucose Tolerance in Severe Obesity
}

\section{Vittoria D'Esposito}

Università degli Studi di Napoli

Maria Rosaria Ambrosio

National Research Council (CNR)

Domenico Liguoro

National Research Council (CNR)

Giuseppe Perruolo

Federico II University of Naples

Manuela Lecce

"Federico II" University of Naples

Serena Cabaro

Federico II University of Naples

Marianna Aprile

National Research Council (CNR)

Ada Marino

Federico II University of Naples

Vincenzo Pilone

Università di Salerno

Pietro Forestieri

Federico II University of Naples

Claudia Miele

CNR - Federico II University of Naples

Dario Bruzzese

Federico II University of Naples

Daniela Terracciano

Federico II University of Naples

Francesco Beguinot

CNR

Pietro Formisano ( $\nabla$ fpietro@unina.it)

"Federico II" University of Naples 
Article

Keywords: subcutaneous adipose tissue, inflammation, glucose tolerance, obesity

Posted Date: February 16th, 2021

DOI: https://doi.org/10.21203/rs.3.rs-123645/v1

License: (c) (i) This work is licensed under a Creative Commons Attribution 4.0 International License. Read Full License

Version of Record: A version of this preprint was published at International Journal of Obesity on May 15th, 2021. See the published version at https://doi.org/10.1038/s41366-021-00850-1. 


\section{Abstract \\ Background}

Excessive adiposity provides an inflammatory environment. However, in people with severe obesity, how systemic and local adipose tissue (AT)-derived cytokines contribute to worsening glucose tolerance is not clear.

\section{Methods}

92 severely obese (SO) individuals undergoing bariatric surgery were enrolled and subjected to detailed clinical phenotyping. Following an Oral Glucose Tolerance Test, participants were included in three groups, based on the presence of normal glucose tolerance (NGT), impaired glucose tolerance (IGT) or Type 2 Diabetes (T2D). Serum and subcutaneous AT (SAT) biopsies were obtained and Mesenchymal Stem Cells (MSCs) were isolated, characterized and differentiated in adipocytes in vitro. TNFA and PPARG mRNA levels were determined by qRT-PCR. Circulating, adipocyte- and MSC-released cytokines, chemokines and growth factors were assessed by multiplex ELISA.

\section{Results}

Serum levels of IL-9, IL-13 and MIP-1 $\beta$ were increased in SO individuals with T2D, as compared with those with either IGT or NGT. At variance, SAT samples obtained from SO individuals with IGT displayed levels of TNFA which were 3-fold higher compared to those with NGT, but not different from those with T2D. Elevated levels of TNFa were also found in differentiated adipocytes, isolated from the SAT specimens of individuals with IGT and T2D, compared to those with NGT. Consistent with the pro-inflammatory milieu, IL-1 $\beta$ and IP-10 secretion was significantly higher in adipocytes from individuals with IGT and T2D. Moreover, increased levels of TNFa, both mRNA and secreted protein, were detected in MSCs obtained from IGT and T2D, compared to NGT SO individuals. Exposure of T2D and IGT-derived MSCs to quercetin reduced TNFa levels and was paralleled by a significant decrease of the secretion of inflammatory cytokines.

\section{Conclusion}

In severe obesity, enhanced SAT-derived inflammatory phenotype is an early step in the progression toward T2D and may be, at least in part, attenuated by quercetin.

\section{Introduction}

Type 2 diabetes (T2D) is a large health and socio-economic burden worldwide. In 2019, about 463 million people were estimated to be living with diabetes and, approximately $90 \%$ of those were affected by T2D. 
This number is expected to increase to 578 million in 2030 and 700 million in $2045^{1}$. The global epidemics of T2D is largely driven by the dramatic increase of obesity and severe obesity observed during the past 35 years ${ }^{2}$. In particular, the prevalence of obesity with Body Mass Index (BMI) $\geq 40 \mathrm{~kg} / \mathrm{m}^{2}$ has quadrupled, and that of obesity with $\mathrm{BMI} \geq 50 \mathrm{~kg} / \mathrm{m}^{2}$ increased fivefold ${ }^{3}$. Recently, it has been shown a $40 \%$ prevalence of T2D in severely obese (SO) population ${ }^{4}$.

Adipose tissue (AT) has a central role in the connection between obesity and T2D. For a long time, AT has been envisioned as a site of energy storage. To date, AT is considered an active endocrine organ, capable of regulating systemic energy and metabolic homeostasis through a complex network of endocrine, paracrine, and autocrine signals. Within AT, mesenchymal stem cells (MSCs) are located in perivascular niches (stromal vascular fraction - SVF) and participate to cell turnover and to the vascular network for AT tropism ${ }^{5,6}$.

Subcutaneous AT (SAT) is by far the largest adipose depot and very relevant for the quantitative contribution to lipid storage/release and endocrine function ${ }^{7}$. Obesity drives the pathological expansion of AT. Unhealthy AT harbours enlarged hypertrophic adipocytes and displays a state of low-grade chronic inflammation. The formation of new vasculature to support AT growth is also impaired, and enhanced fibrosis and hypoxia are evident. Such events contribute to the development of insulin resistance ${ }^{8}$. Dysfunctional AT secretes pro-inflammatory cytokines that antagonize insulin action, promoting local and systemic inflammation 7,8 . For instance, tumor necrosis factor- a (TNF-a) plays a well-known role in the connection between obesity, inflammation and insulin resistance ${ }^{9}$.

Thus, although excess weight is an established risk factor for T2D, yet a large part of obese individuals do not develop T2D and the precise mechanisms linking the two conditions remain unclear ${ }^{10,11}$. Moreover, there are still few studies focused on the progression toward T2D in SO individuals ${ }^{4}$.

To clarify whether AT-related low-grade inflammation may contribute to metabolic derangement in severe obesity, we enrolled a cohort of SO individuals undergoing bariatric surgery, with or without impairment of glucose tolerance. We found that SAT adipocytes and their precursor MSCs derived from SO individuals with abnormal glucose tolerance, displayed increased production of inflammatory molecules compared to those with normal glucose tolerance (NGT). Deranged production of cytokines by MSCs was at least in part reverted by the flavonoid quercetin.

\section{Material And Methods}

\section{Patient enrollment and tissue collection.}

Abdominal human SAT biopsies and serum samples were obtained from $\mathrm{N}=92$ subjects undergoing laparoscopic gastric banding from October 2013 to April 2015. Exclusion criteria were: metabolic and endocrine diseases, gastrointestinal inflammatory diseases, previous or current malignancies. All patients enrolled underwent detailed clinical phenotyping, including measurement of height, weight, waist, blood 
pressure, complete clinical chemistry, inflammatory and hormone determination (thyroid, adrenal and sexual). Moreover, patients were subjected to an Oral Glucose Tolerance Test (OGTT) with the administration of $75 \mathrm{~g}$ of glucose and blood drawn at 0,30, 60, 90 and $120 \mathrm{~min}$ for plasma glucose and insulin determination. IGT and T2D were diagnosed based on American Diabetes Association criteria ${ }^{12}$. Informed consent was obtained from every subject before the surgical procedure. Protocols were approved by the ethical committee of the University of Naples (prot. n. 225_2013).

\section{Cell isolation and differentiation.}

Mesenchymal stem cells (MSCs) were isolated from SAT biopsies as previously described ${ }^{13}$. Briefly, SAT specimens were chopped into small pieces and were enzymatically digested by collagenase solution ( $1 \mathrm{mg} / \mathrm{ml}$ - Sigma-Aldrich, St. Louis, MO, USA) for $1 \mathrm{~h}$ at 37C. The stromal vascular fraction (SVF) containing MSCs was obtained by centrifugation at $1200 \mathrm{~g}$ for 5 minutes and plated in regular DMEM (Dulbecco's modified Eagle's medium) - F12 (1:1) supplemented with 10\% fetal bovine serum (FBS), 2 mM glutamine, 100 units/ml penicillin and 100 units/ml streptomycin (Lonza, Group Ltd, Basel, Switzerland). At passage 4, MSCs were characterized for the presence of mesenchymal progenitor cell surface antigens CD29 and CD73, for the absence of CD106 and CD45 and for their ability to undergo to adipogenic differentiation (Suppl. Figure 1), as indicated ${ }^{14}$. Adipogenic differentiation and Oil Red 0 staining and detection, to assess lipid accumulation, were performed as previously reported ${ }^{15}$.

\section{Determination of cytokines, chemokines and growth factors.}

Serum samples and cell supernatants were screened for the concentration of interleukin (IL)-1 ra, IL-1b, IL2, IL-4, IL-5, IL-6, IL-7, IL-8, IL-9, IL-10, IL-12(p70), IL-13, IL-15, IL-17A, basic Fibroblast Growth Factor (FGF), Eotaxin, Granulocyte- Colony Stimulating Factor (G-CSF), Granulocyte-Macrophage Colony-Stimulating Factor (GM-CSF), Interferon-y (IFN-y), Interferon- $\gamma$ inducible Protein 10 (IP-10), Monocyte Chemoattractant Protein-1 (MCP-1), Macrophage Inflammatory Protein-1 (MIP-1) a, MIP-1 $\beta$, C-C motif Chemokine ligand 5 (CCL5)/RANTES, TNF-a, Platelet derived Growth Factor (PDGF-BB) and Vascular Endothelial Growth Factor (VEGF) using the Bioplex multiplex Human Cytokine, Chemokine and Growth factor kit (Bio-Rad, Hercules, CA, USA). Ghrelin, Gastric Inhibitory Peptide (GIP), Glucagon-like peptide-1 (GLP-1) and Glucagon concentration was determined by using the Bioplex multiplex Human Diabetes kit, according to the manufacturer's protocol, as previously described ${ }^{16,17}$. The magnetic Bead-Based Assay was performed on a Bio-Plex 200 System (Bio-Rad, Hercules, CA, USA).

\section{RNA isolation and Quantitative RT- PCR.}

Total RNA was isolated from cells and tissues using TRIzol solution (Life Technologies, Carlsbad, CA, USA) as previously reported ${ }^{18}$. RNA was reverse transcribed using SuperScript III Reverse Transcriptase (Life Thechnologies, CarlsBad, CA). qRT-PCR was carried out by using SYBR Green mix (Bio-Rad, Hercules, CA). Reactions were performed using Platinum SYBR Green Quantitative PCR Super- UDG using an iCycler IQ multicolor Real-time PCR Detection System (Bio-Rad, Hercules, CA). All reactions were 
performed in duplicate and Peptidylprolyl Isomerase A - PPIA was used as an internal standard. Primer sequences for PPIA, TNFA, canonical PPARG are the following: PPIA 5' TACGGGTCCTGGCATCTTGT 3'- 5' GGTGATCTTCTTGCTGGTCT 3'; TNFA 5' GGCTTGTCACTCGGGGTT 3' - 5' GGGACCTCTCTCTAATCAGCC 3'; PPARG 5' GAGAAGGAGAAGCTGTTGGC 3' - 5' ATGGCCACCTCTTTGCTCT 3'.

\section{Statistical Analysis.}

Statistical analyses were performed with R statistical platform and with GraphPad Prism 6.0 software (GraphPad Software Inc., La Jolla, CA). Results are presented as mean \pm standard deviation (SD) for continuous variables if normally distributed and as median and range if not. For comparisons between 2 groups, a two-tailed t-test for independent samples (for normally distributed data) or a Mann-Whitney U test (for not normally distributed data) was used. Multiple comparisons among the three groups were made using the ANOVA test with Tukey correction (for normally distributed data) or the Kruskal-Wallis test followed by the Dunn test (for not normally distributed data). $p$ value of $<0.05$ were considered statistically significant.

\section{Results}

\section{Anthropometric and clinical characteristics of the study population}

Severely obese (SO) individuals enrolled in the study ( $\mathrm{N}=92)$ were subjected to OGTT and grouped as NGT ( $=44)$, IGT $(\mathrm{N}=25)$ and T2D $(\mathrm{N}=23)$. NGT, IGT and T2D groups did not display significant differences for mean age, BMI, abdominal circumference, arm circumference, systolic and diastolic blood pressure (Table 1). HOMA-IR was significantly higher in T2D patients compared to IGT and NGT (Table 1). No differences in serum levels of the gastro-intestinal hormones Ghrelin, GIP, GLP-1 and Glucagon were found among the 3 groups (Suppl. Table 1). Finally, NGT, IGT and T2D groups had similar concentrations of pituitary, thyroid and sexual hormones (data not shown).

\section{Systemic inflammation in NGT, IGT and T2D subjects}

Serum samples of the 3 groups of patients were screened for the concentration of C-reactive Protein (CRP), Fibrinogen and Ferritin, as classical markers of systemic inflammation and no significant differences were identified among the 3 groups of SO subjects (Suppl. Table 2). However, the screening of a panel of cytokines, chemokines and growth factors revealed that T2D patients displayed an increase of IL-9, IL-13 and MIP-1 $\beta$ compared to IGT (1.2, 1.4, 1.5-fold, respectively) and of IL-13 compared to NGT (1.5-fold). Thus, higher levels of specific cytokines characterize the serum of T2D SO subjects. Moreover, no significant difference was found between IGT and NGT groups, beside a slight reduction of IL-9 levels in SO individuals with IGT (Table 2).

\section{Adipose tissue inflammation in NGT, IGT and T2D SO subjects}


In order to assess whether subcutaneous adipose tissue (SAT) may contribute to the worsening of glucose tolerance in SO individuals, SAT biopsies from a subset of patients were collected and analyzed for quantification of specific mRNAs. As shown in fig. 1A, no differences in mRNA levels of the canonical isoforms of adipogenic master gene PPARG were found. However, SAT from IGT and T2D patients displayed a 3-fold increased mRNA levels of the inflammatory marker TNF-a, compared with NGT (fig. 1B).

Next, SAT-derived mesenchymal stem cells (MSCs) were isolated and characterized, as described in Materials and Methods and in Supplementary figure 1. MSCs from the 3 groups displayed the same ability to differentiate in adipocytes (Suppl. fig. 2). However, adipocytes obtained from IGT SO patients released higher levels of TNF- $a$ (pval= 0.01 ), IL-1 $(p v a l=0.04)$ and IP-10 (pval=0.08) compared to NGT SO patients (fig. 2). TNF-a, IL-1 $\beta$ and IP-10 secretion was significantly higher also in adipocytes from T2D compared with those of NGT subjects (fig.3). No detectable differences between T2D and IGT were identified (fig. 2).

Next, in order to assess whether the inflammatory phenotype of adipocytes was already displayed by their mesenchymal precursor cells, TNF-a levels were evaluated in MSCs obtained from the 3 groups. Interestingly, significantly increased TNF-a mRNA and protein levels were observed in MSCs from IGT, compared to NGT patients, with no significant differences between IGT and T2D (fig. 3). Thus, SAT precursor MSCs from SO subjects with abnormal glucose tolerance are primed to develop an inflammatory phenotype.

\section{Quercetin reduces MSC inflammation in SO subjects}

Finally, we investigated whether the flavonoid quercetin was able to counteract MSC inflammation in IGT and T2D SO subjects. Quercetin treatment did not impair MSC viability (data not shown). However, quercetin exposure reduced TNFA mRNA levels by about 3-fold in MSCs from IGT subjects (fig. 4A). In addition, it significantly reduced TNF-a protein secretion in MSCs both from IGT and from T2D subjects (fig. 4B). Interestingly, in presence of quercetin a significant decrease of the inflammatory cytokines IL-4, IL-6, IL-7, INF- $\gamma$ and MCP-1 in IGT and of IL-1 $\beta$, IL-4, IL-6, IL-7, INF- $y$, MCP-1 and FGFb in T2D-MSCs was also observed (fig. 4B). Quercetin did not modify the release of IL2, IL-17, CCL5, MIP-1a, MIP-1 $\beta$ and IP-10 both in IGT and T2D derived MSCs (data not shown). Notably, quercetin treatment induced a significant increase of PDGF and G-CSF in MSCs of IGT and T2D SO subjects (fig. 4B). Thus, quercetin is able to modify inflammatory signals in SAT precursor cells.

\section{Discussion}

Obesity is a clear risk factor for metabolic, cardiovascular and oncological diseases ${ }^{2,5}$. In particular, the prevalence of T2D increases with increasing BMI. Hence, SO subjects have a higher risk than moderately obese individuals ${ }^{19}$. However, there are $7-28.3 \%$ SO subjects, who do not develop T2D ${ }^{20}$. Most of the 
studies investigated the metabolic abnormalities associated with the more common moderate obesity, while much fewer data is available for morbid obese patients ${ }^{4}$.

Here, we have shown, for the first time, that in SO subjects: i) circulating IL-9, IL-13 and MIP-1 $\beta$ are associated to T2D; ii) in the progression of glucose tolerance impairment, inflammation is detectable in SAT earlier than in serum; iii) SAT-derived MSCs from IGT are already educated to secrete more TNF-a; iiii) quercetin is able to control MSC inflammation.

Obesity and T2D are characterized by systemic low-grade inflammation with higher levels of both proinflammatory and anti-inflammatory cytokines ${ }^{10,21-23}$. However, conflicting data and discrepancies about the composition of the cytokinome exist. First, we have observed that SO subjects affected by T2D displayed higher levels of serum IL-9, IL-13 and MIP-1 $\beta$, as compared to IGT.

IL-9 is a pleiotropic inflammatory cytokine also implicated in pancreatic $\beta$-cell destruction in T1D 24, 25 . MIP-1 $\beta$, also known as chemokine CC motif ligand 4 -CCL4, is a potent macrophage attractant, which acts through the binding to CCR5, a transmembrane receptor involved in infection, inflammation and cancer ${ }^{26}$. At variance, IL-13 is an anti-inflammatory cytokine that promotes polarization of macrophages toward a M2 phenotype: it contributes to epicardial adipose tissue remodeling and increases glucose uptake and metabolism in skeletal muscle ${ }^{27,28}$. Previous studies have detected increased levels of IL-9, IL-13 and MIP-1 $\beta$ in serum of obese individuals compared with lean controls ${ }^{21-23}$. Other studies in T2D have also reported an increase of MIP-1 $\beta$, a reduction or no change of IL-13 and no difference of IL-9 compared to NGT subjects $21,23,26,27,29$. However, at our knowledge, no data are available for T2D and IGT morbid obese individuals. In this population, the increase of IL-9 and MIP-1 $\beta$ is consistent with their possible involvement in $\beta$-cell damage and macrophage recruitment, respectively, and contribute to the progression from IGT toward T2D. On the other side, IL-13 increase may be due to a compensatory mechanism. Indeed, all T2D individuals enrolled in the study were either newly diagnosed or with a short duration of disease, also for their relatively young age. Moreover, no significant differences were detected in the systemic levels of cytokines of SO individuals with IGT, as compared to those with NGT.

AT has a central role in the association between obesity and T2D and is now considered a prominent target for new pharmacological approaches, including adipokine-based therapeutics ${ }^{8}$. Excess glucose, lipids and other nutrients lead to an impaired AT with altered plasticity and imbalanced production of adipokines and pro-inflammatory factors, which sustain a local low-grade inflammation that is a crucial determinant for T2D and obesity complications ${ }^{5,11,30}$. We have provided evidence that SAT and SATderived isolated cells of SO IGT and T2D patients expressed significantly higher levels of TNF-a compared to NGT patients, with no detectable differences between T2D and IGT. TNF- $a$ is a well-known inflammatory cytokine, with a pivotal role in the onset and progression of insulin resistance ${ }^{9}$. TNF-a levels are increased in AT and plasma of obese individuals, and its expression is positively related to BMI with no significant relationship between fat cell size and mRNA levels ${ }^{9,31-33}$. In addition to TNF- $a$, we have found that adipocytes from IGT SO people released higher levels of IL-1 $\beta$ and IP-10, other key 
cytokines in obesity and in etiology of T2D ${ }^{34}$. TNFa and IL-1 $\beta$ work together to both initiate and propagate the inflammatory process, also inducing the expression of multiple cytokines, among which IP$10{ }^{35,36}$. IP-10, also known as CXCL10, has been shown to play a deleterious role in obesity as potential inhibitor of AT angiogenesis ${ }^{37}$. Thus, our data suggest that TNF-a, IL-1 $\beta$ and IP-10 are not only adipocytokines associated to obesity, but also molecules with a crucial role in the early phases of impairment of glucose metabolism in SO subjects.

SAT from obese subjects contains a dysfunctional pool of MSCs with impaired adipogenesis ${ }^{38,39}$. We have previously shown that a truncated isoform of PPAR (PPAR $\triangle 5$ ), which acts as a dominant-negative isoform by reducing PPARY activity, positively correlates - whereas canonical PPARG does not - with BMI in overweight or obese and T2D patients, possibly contributing to impaired adipogenesis ${ }^{40}$. Furthermore, an increased ratio between PPAR 5 and canonical isoforms has been recently reported in a in vitro generated model of hypertrophic-like adipocytes, also displaying increasing IL-6 secretion, whereas only a modest reduction of canonical PPARG expression was observed ${ }^{41}$. Here, we detected similar levels of canonical PPARG in SAT biopsies of SO individuals with different glucose tolerance conditions, as well as similar levels of lipid accumulation in differentiated adipocytes. Thus, most likely, the difference in cytokine release does not relate to a different adipocyte differentiation program.

Notably, we found that MSCs from IGT subjects are already primed to express and secrete more TNFa, compared to MSCs from NGT subjects. High glucose exposure of MSCs leads to impaired cell functions, upregulation of inflammatory genes (IL-1 $\beta$, CCL5, IL-8, MCP-1) and alteration in DNA methylation 38, 42, 43 . Epigenetic modifications represent a common mechanism through which both genetic and environmental exposures impact on the susceptibility to T2D ${ }^{44}$. It has been shown that distinct methylations of the promoter regulate TNF-a expression ${ }^{45,46}$. It could be hypothesized that impaired glucose levels may imprint specific epigenetic signature on MSCs, upregulating TNF-a production, which in turn contributes to impaired glucose tolerance. However, further studies are necessary to elucidate the molecular mechanisms underlining increased TNF-a levels in IGT- derived MSCs.

To date, a special attention for both prophylactic and therapeutic interventions in T2D is directed to diet nutrients, able to regulate the metabolism and influence the consumer's health ${ }^{47}$. Quercetin is a nutraceutical compound with anti-inflammatory and antioxidant effects both in vivo and in vitro. Possible mechanisms of actions include inhibition of COX-2, NF-kB and MAPK pathways, post-translational modifications and elimination of senescent cells ${ }^{47-49}$. Here, we have shown that quercetin ameliorates the local low-grade inflammation by reducing the expression of TNF-a and the release of a number of inflammatory cytokines in MSCs isolated from IGT and T2D morbid obese subjects. Moreover, it increases the release of G-CSF, a cytokine able to counteract the activation of major inflammatory cytokines, including TNF-a, whose anti-obesity effects have been shown in animal models with diabetes and obesity ${ }^{50}$.

\section{Conclusions}


Specific patterns of circulating and AT-released cytokines may have a crucial role in the worsening of glucose tolerance allowing the identification of subjects with unhealthy obesity that could develop T2D. Of course, many other factors may contribute to the progression from normal glucose tolerance to impaired glucose tolerance in SO subjects. For instance, even though abdominal SAT contributes more than visceral AT (VAT) to the pro-inflammatory milieu associated with SO ${ }^{51}$, VAT- derived cytokines, whose release correlates with epigenetic modifications in SO-VAT ${ }^{44}$, may also play a relevant role, and thus require further investigations.

\section{Declarations}

\section{Acknowledgements}

The authors wish to thank Dr. Virginia Tagliamonte, Dr. Vincenzo Cosimato and Dr. Aniello Rainone involved in patient enrollment and clinical phenotyping and in the management of the clinical records.

\section{Competing Interests}

The authors declare no competing financial interests

\section{Funding}

This work was supported by the Regione Campania POR FESR 2014-2020-Objective 1.2.-Realization of Technology Platform to fight oncologic diseases (RARE PLAT NET, SATIN, and COEPICA Projects) and by the Italian Association for the Cancer Research-AIRC (grant IG19001).

\section{Author contributions}

Vittoria D’Esposito: Conceptualization, Investigation, Data curation, Formal analysis, Writing - Original Draft, Writing - Review \& Editing. Maria Rosaria Ambrosio: Conceptualization, Investigation, Data curation. Domenico Liguoro: Investigation, Methodology. Giuseppe Perruolo: Investigation. Manuela Lecce: Investigation, Formal analysis. Serena Cabaro: Investigation, Writing - review \& editing. Marianna Aprile: Investigation. Ada Marino: Data curation. Vincenzo Pilone: Investigation. Pietro Forestieri: Conceptualization, Supervision. Claudia Miele: Funding acquisition, Supervision. Dario Bruzzese: Data curation, Formal analysis. Daniela Terracciano: Project administration, Supervision. Francesco Beguinot: Funding acquisition, Resources. Pietro Formisano: Conceptualization; Funding acquisition; Project administration; Resources; Supervision, Writing - original draft; Writing - review \& editing.

\section{References}

1. Saeedi P, Petersohn I, Salpea P, Malanda B, Karuranga S, Unwin N et al. Global and regional diabetes prevalence estimates for 2019 and projections for 2030 and 2045: Results from the International Diabetes Federation Diabetes Atlas, 9(th) edition. Diabetes research and clinical practice 2019; 157: 107843. 
2. Chooi YC, Ding C, Magkos F. The epidemiology of obesity. Metabolism: clinical and experimental 2019; 92: 6-10.

3. Schernthaner G, Morton JM. Bariatric surgery in patients with morbid obesity and type 2 diabetes. Diabetes Care 2008; 31 Suppl 2: S297-302.

4. Silveira EA, de Souza Rosa LP, de Carvalho Santos A, de Souza Cardoso CK, Noll M. Type 2 Diabetes Mellitus in Class II and III Obesity: Prevalence, Associated Factors, and Correlation between Glycemic Parameters and Body Mass Index. International journal of environmental research and public health 2020; 17(11).

5. D'Esposito V, Ambrosio MR, Giuliano M, Cabaro S, Miele C, Beguinot F et al. Mammary Adipose Tissue Control of Breast Cancer Progression: Impact of Obesity and Diabetes. Frontiers in oncology 2020; 10: 1554.

6. Poulos SP, Hausman DB, Hausman GJ. The development and endocrine functions of adipose tissue. Molecular and cellular endocrinology 2010; 323(1): 20-34.

7. Smith U, Kahn BB. Adipose tissue regulates insulin sensitivity: role of adipogenesis, de novo lipogenesis and novel lipids. Journal of internal medicine 2016; 280(5): 465-475.

8. Kusminski CM, Bickel PE, Scherer PE. Targeting adipose tissue in the treatment of obesity-associated diabetes. Nature reviews. Drug discovery 2016; 15(9): 639-660.

9. Hotamisligil GS, Spiegelman BM. Tumor necrosis factor alpha: a key component of the obesitydiabetes link. Diabetes 1994; 43(11): 1271-8.

10. Eckel RH, Kahn SE, Ferrannini E, Goldfine AB, Nathan DM, Schwartz MW et al. Obesity and type 2 diabetes: what can be unified and what needs to be individualized? Diabetes care $2011 ; 34(6)$ : 142430 .

11. Zatterale F, Longo M, Naderi J, Raciti GA, Desiderio A, Miele C et al. Chronic Adipose Tissue Inflammation Linking Obesity to Insulin Resistance and Type 2 Diabetes. Frontiers in physiology 2019; 10: 1607.

12. American Diabetes A. Diagnosis and classification of diabetes mellitus. Diabetes care 2014; 37 Suppl 1: S81-90.

13. D'Esposito V, Lecce M, Marenzi G, Cabaro S, Ambrosio MR, Sammartino G et al. Platelet-rich plasma counteracts detrimental effect of high-glucose concentrations on mesenchymal stem cells from Bichat fat pad. Journal of tissue engineering and regenerative medicine 2020; 14(5): 701-713.

14. de Girolamo L, Lucarelli E, Alessandri G, Avanzini MA, Bernardo ME, Biagi E et al. Mesenchymal stem/stromal cells: a new "cells as drugs" paradigm. Efficacy and critical aspects in cell therapy. Current pharmaceutical design 2013; 19(13): 2459-73.

15. Aprile M, Ambrosio MR, D'Esposito V, Beguinot F, Formisano P, Costa V et al. PPARG in Human Adipogenesis: Differential Contribution of Canonical Transcripts and Dominant Negative Isoforms. PPAR research 2014; 2014: 537865.

16. Caso F, Postiglione L, Covelli B, Ricciardone M, Di Spigna G, Formisano P et al. Pro-inflammatory adipokine profile in psoriatic arthritis: results from a cross-sectional study comparing PsA subset 
with evident cutaneous involvement and subset "sine psoriasis". Clinical rheumatology 2019; 38(9): 2547-2552.

17. Pisano S, Catone G, Coppola G, Carotenuto M, Iuliano R, Tiano C et al. Different Immune Signature in Youths Experiencing Antipsychotic-Induced Weight Gain Compared to Untreated Obese Patients. Journal of child and adolescent psychopharmacology 2017; 27(9): 844-848.

18. Ambrosio MR, D'Esposito V, Costa V, Liguoro D, Collina F, Cantile M et al. Glucose impairs tamoxifen responsiveness modulating connective tissue growth factor in breast cancer cells. Oncotarget 2017; 8(65): 109000-109017.

19. Vinciguerra F, Baratta R, Farina MG, Tita P, Padova G, Vigneri R et al. Very severely obese patients have a high prevalence of type 2 diabetes mellitus and cardiovascular disease. Acta diabetologica 2013; 50(3): 443-9.

20. Catoi AF, Busetto L. Metabolically Healthy Obesity and Bariatric Surgery. Obesity surgery 2019; 29(9): 2989-3000.

21. Dalmas E, Rouault C, Abdennour M, Rovere C, Rizkalla S, Bar-Hen A et al. Variations in circulating inflammatory factors are related to changes in calorie and carbohydrate intakes early in the course of surgery-induced weight reduction. The American journal of clinical nutrition 2011; 94(2): 450-8.

22. Schmidt FM, Weschenfelder J, Sander C, Minkwitz J, Thormann J, Chittka T et al. Inflammatory cytokines in general and central obesity and modulating effects of physical activity. PloS one 2015; 10(3): e0121971.

23. van der Zalm IJB, van der Valk ES, Wester VL, Nagtzaam NMA, van Rossum EFC, Leenen PJM et al. Obesity-associated T-cell and macrophage activation improve partly after a lifestyle intervention. International journal of obesity 2020; 44(9): 1838-1850.

24. Chakraborty S, Kubatzky KF, Mitra DK. An Update on Interleukin-9: From Its Cellular Source and Signal Transduction to Its Role in Immunopathogenesis. International journal of molecular sciences 2019; 20(9).

25. Stechova K, Halbhuber Z, Hubackova M, Kayserova J, Petruzelkova L, Vcelakova J et al. Case report: type 1 diabetes in monozygotic quadruplets. European journal of human genetics: EJHG 2012; 20(4): 457-62.

26. Chang TT, Chen JW. Emerging role of chemokine CC motif ligand 4 related mechanisms in diabetes mellitus and cardiovascular disease: friends or foes? Cardiovascular diabetology 2016; 15(1): 117.

27. Jiang LQ, Franck N, Egan B, Sjogren RJ, Katayama M, Duque-Guimaraes D et al. Autocrine role of interleukin-13 on skeletal muscle glucose metabolism in type 2 diabetic patients involves microRNA let-7. American journal of physiology. Endocrinology and metabolism 2013; 305(11): E1359-66.

28. Parisi V, Cabaro S, D'Esposito V, Petraglia L, Conte M, Campana P et al. Epicardial Adipose Tissue and IL-13 Response to Myocardial Injury Drives Left Ventricular Remodeling After ST Elevation Myocardial Infarction. Frontiers in physiology 2020; 11: 575181.

29. Chen H, Wen F, Zhang X, Su SB. Expression of T-helper-associated cytokines in patients with type 2 diabetes mellitus with retinopathy. Molecular vision 2012; 18: 219-26. 
30. Crewe C, An YA, Scherer PE. The ominous triad of adipose tissue dysfunction: inflammation, fibrosis, and impaired angiogenesis. The Journal of clinical investigation 2017; 127(1): 74-82.

31. Bullo M, Garcia-Lorda P, Peinado-Onsurbe J, Hernandez M, Del Castillo D, Argiles JM et al. TNFalpha expression of subcutaneous adipose tissue in obese and morbid obese females: relationship to adipocyte LPL activity and leptin synthesis. International journal of obesity and related metabolic disorders: journal of the International Association for the Study of Obesity 2002; 26(5): 652-8.

32. Hoffstedt J, Arner E, Wahrenberg H, Andersson DP, Qvisth V, Lofgren P et al. Regional impact of adipose tissue morphology on the metabolic profile in morbid obesity. Diabetologia 2010; 53(12): 2496-503.

33. Katsuki A, Sumida Y, Murashima S, Murata K, Takarada Y, Ito K et al. Serum levels of tumor necrosis factor-alpha are increased in obese patients with noninsulin-dependent diabetes mellitus. The Journal of clinical endocrinology and metabolism 1998; 83(3): 859-62.

34. Febbraio MA. Role of interleukins in obesity: implications for metabolic disease. Trends in endocrinology and metabolism: TEM 2014; 25(6): 312-9.

35. Narumi S, Yoneyama H, Inadera H, Nishioji K, Itoh Y, Okanoue T et al. TNF-alpha is a potent inducer for IFN-inducible protein-10 in hepatocytes and unaffected by GM-CSF in vivo, in contrast to IL-1beta and IFN-gamma. Cytokine 2000; 12(7): 1007-16.

36. Parisi V, Petraglia L, Cabaro S, D'Esposito V, Bruzzese D, Ferraro G et al. Imbalance Between Interleukin-1 beta and Interleukin-1 Receptor Antagonist in Epicardial Adipose Tissue Is Associated With Non ST-Segment Elevation Acute Coronary Syndrome. Frontiers in physiology 2020; 11: 42.

37. Hueso L, Ortega R, Selles F, Wu-Xiong NY, Ortega J, Civera M et al. Upregulation of angiostatic chemokines IP-10/CXCL10 and I-TAC/CXCL11 in human obesity and their implication for adipose tissue angiogenesis. International journal of obesity 2018; 42(8): 1406-1417.

38. Ejarque M, Ceperuelo-Mallafre V, Serena C, Maymo-Masip E, Duran X, Diaz-Ramos A et al. Adipose tissue mitochondrial dysfunction in human obesity is linked to a specific DNA methylation signature in adipose-derived stem cells. International journal of obesity 2019; 43(6): 1256-1268.

39. Longo M, Zatterale F, Naderi J, Parrillo L, Formisano P, Raciti GA et al. Adipose Tissue Dysfunction as Determinant of Obesity-Associated Metabolic Complications. International journal of molecular sciences 2019; 20(9).

40. Aprile M, Cataldi S, Ambrosio MR, D'Esposito V, Lim K, Dietrich A et al. PPARgammaDelta5, a Naturally Occurring Dominant-Negative Splice Isoform, Impairs PPARgamma Function and Adipocyte Differentiation. Cell reports 2018; 25(6): 1577-1592 e6.

41. Aprile M, Cataldi S, Perfetto C, Ambrosio MR, Italiani P, Tate R et al. In-Vitro-Generated HypertrophicLike Adipocytes Displaying PPARG Isoforms Unbalance Recapitulate Adipocyte Dysfunctions In Vivo. Cells 2020; 9(5).

42. D'Esposito V, Passaretti F, Hammarstedt A, Liguoro D, Terracciano D, Molea G et al. Adipocytereleased insulin-like growth factor-1 is regulated by glucose and fatty acids and controls breast cancer cell growth in vitro. Diabetologia 2012; 55(10): 2811-2822. 
43. Ronningen $\mathrm{T}$, Shah $\mathrm{A}$, Reiner $\mathrm{AH}$, Collas $\mathrm{P}$, Moskaug JO. Epigenetic priming of inflammatory response genes by high glucose in adipose progenitor cells. Biochemical and biophysical research communications 2015; 467(4): 979-86.

44. Raciti GA, Spinelli R, Desiderio A, Longo M, Parrillo L, Nigro C et al. Specific CpG hyper-methylation leads to Ankrd26 gene down-regulation in white adipose tissue of a mouse model of diet-induced obesity. Scientific reports 2017; 7: 43526.

45. Gowers IR, Walters K, Kiss-Toth E, Read RC, Duff GW, Wilson AG. Age-related loss of CpG methylation in the tumour necrosis factor promoter. Cytokine 2011; 56(3): 792-7.

46. Rancourt RC, Ott R, Ziska T, Schellong K, Melchior K, Henrich W et al. Visceral Adipose Tissue Inflammatory Factors (TNF-Alpha, SOCS3) in Gestational Diabetes (GDM): Epigenetics as a Clue in GDM Pathophysiology. International journal of molecular sciences 2020; 21(2).

47. Nettore IC, Rocca C, Mancino G, Albano L, Amelio D, Grande F et al. Quercetin and its derivative Q2 modulate chromatin dynamics in adipogenesis and Q2 prevents obesity and metabolic disorders in rats. The Journal of nutritional biochemistry 2019; 69: 151-162.

48. Chen S, Jiang H, Wu X, Fang J. Therapeutic Effects of Quercetin on Inflammation, Obesity, and Type 2 Diabetes. Mediators of inflammation 2016; 2016: 9340637.

49. Palmer AK, Xu M, Zhu Y, Pirtskhalava T, Weivoda MM, Hachfeld CM et al. Targeting senescent cells alleviates obesity-induced metabolic dysfunction. Aging cell 2019; 18(3): e12950.

50. Lee Y, Song YS, Fang CH, So BI, Park JY, Joo HW et al. Anti-obesity effects of granulocyte-colony stimulating factor in Otsuka-Long-Evans-Tokushima fatty rats. PloS one 2014; 9(8): e105603.

51. Spoto B, Di Betta E, Mattace-Raso F, Sijbrands E, Vilardi A, Parlongo RM et al. Pro- and antiinflammatory cytokine gene expression in subcutaneous and visceral fat in severe obesity. Nutrition, metabolism, and cardiovascular diseases: NMCD 2014; 24(10): 1137-43.

\section{Tables}

\begin{tabular}{lcccc}
\hline & NGT $(\mathrm{n}=44)$ & IGT $(\mathrm{n}=25)$ & T2DM $(\mathrm{n}=23)$ & Overall p value \\
\hline Gender; Female & $35(79.5)$ & $17(70.8)$ & $14(60.9)$ & 0.259 \\
Age & $34.1 \pm 10.8$ & $37.4 \pm 10$ & $40 \pm 10.4$ & 0.091 \\
BMI & $43.4[40.4 ; 46.6]$ & $41.5[39.9 ; 51.7]$ & $45[41 ; 51.8]$ & 0.401 \\
Waist Circ. (cm) & $132[121 ; 142]$ & $135[123.8 ; 143]$ & $138.5[125.5 ; 151.3]$ & 0.320 \\
Arm Circ. (cm) & $40[38 ; 44]$ & $40[39 ; 46.9]$ & $43[39 ; 47.3]$ & 0.184 \\
Syst. bl. Press. (mmHg) & $125[120 ; 140]$ & $130[127.5 ; 130]$ & $122.5[120 ; 135]$ & 0.478 \\
Diast. bl. Press. (mmHg) & $80[80 ; 90]$ & $80[70 ; 86.3]$ & $80[78.5 ; 85]$ & 0.744 \\
HOMA-IR & $2.4[1.5 ; 3.3]$ & $3.6[2.6 ; 5.1]$ & $6.8[4.7 ; 11]$ & $<0.001$ \\
\hline
\end{tabular}

Table 1. Clinical phenotyping of the population enrolled ( $N=92$ ). Gender (female) is expressed as number (percentage); age is expressed as mean \pm SD. Other data are expressed as median and range [min; max]. 


\begin{tabular}{|c|c|c|c|c|c|c|c|}
\hline & $\begin{array}{l}\text { NGT } \\
\mathrm{pg} / \mathrm{ml}\end{array}$ & $\begin{array}{l}\text { IGT } \\
\mathrm{pg} / \mathrm{ml}\end{array}$ & $\begin{array}{l}\text { T2D } \\
\mathrm{pg} / \mathrm{ml}\end{array}$ & $\begin{array}{l}\text { Overall p- } \\
\text { value }\end{array}$ & $\begin{array}{l}\text { IGT vs. } \\
\text { NGT }\end{array}$ & $\begin{array}{l}\text { T2D vs } \\
\text { IGT }\end{array}$ & $\begin{array}{l}\text { T2D vs } \\
\text { NGT }\end{array}$ \\
\hline IL-1 $\beta$ & $5[4.6 ; 6.3]$ & $5.1[4.2 ; 6.1]$ & $5.1[4.6 ; 6]$ & 0.851 & & & \\
\hline IL-1ra & $\begin{array}{l}349.9 \text { [325.8; } \\
370.8]\end{array}$ & $357.2[305 ; 410.4]$ & $356.9[279.8 ; 446.8]$ & 0.926 & & & \\
\hline IL-2 & $45.7[35.3 ; 48.9]$ & $41.5[1.7 ; 50.8]$ & $40.1[21 ; 46]$ & 0.280 & & & \\
\hline IL-4 & $5.7[4.8 ; 8.5]$ & $5.5[4.1 ; 9.9]$ & $7.4[5.1 ; 10.3]$ & 0.338 & & & \\
\hline $\mathrm{IL}-5$ & $3.8[3.4 ; 8.5]$ & $5[3.6 ; 10.9]$ & $7.7[4 ; 11.5]$ & 0.111 & & & \\
\hline IL-6 & $22.7[19.2 ; 27.8]$ & $24.1[17.1 ; 28.2]$ & $25.8[20 ; 30.7]$ & 0.728 & & & \\
\hline $\mathrm{IL}-7$ & $15[12.4 ; 22.8]$ & $16.4[11.8 ; 27.2]$ & $22.5[13.8 ; 35.7]$ & 0.206 & & & \\
\hline $\mathrm{IL}-8$ & $66.4[42.3 ; 92.9]$ & $54[41.2 ; 70.9]$ & $48.6[41.5 ; 104.2]$ & 0.576 & & & \\
\hline $\mathrm{IL}-9$ & $45.4[39 ; 68.9]$ & $40.2[25.7 ; 48]$ & $50.6[42.5 ; 70.6]$ & 0.004 & 0.03 & 0.006 & 1 \\
\hline IL-10 & $20.7[17.1 ; 28.2]$ & $19.3[8.6 ; 30.8]$ & $29.2[17.2 ; 39.3]$ & 0.088 & & & \\
\hline $\begin{array}{l}\mathrm{IL}- \\
12 \mathrm{p} 70\end{array}$ & $101.6[76.2 ; 133.3]$ & $88.3[45.3 ; 121.7]$ & $102[89.3 ; 142.2]$ & 0.174 & & & \\
\hline IL-13 & $11.4[9.3 ; 16.6]$ & $12.3[8.2 ; 18.5]$ & $17.1[10.7 ; 31.3]$ & 0.027 & 1 & 0.045 & 0.039 \\
\hline IL-15 & $56.5[53.6 ; 64.3]$ & $64.5[57.4 ; 72.5]$ & $64.2[55.5 ; 71]$ & 0.053 & & & \\
\hline IL-17 & $\begin{array}{l}136.8[119.5 ; \\
210.3]\end{array}$ & $127.8[90.2 ; 222.1]$ & $208.9[132.6 ; 258.9]$ & 0.030 & & & \\
\hline Eotaxin & $134.2[103 ; 289.2]$ & $112.6[44.9 ; 409.4]$ & $170.6[98.1 ; 393.9]$ & 0.471 & & & \\
\hline G-CSF & $\begin{array}{l}134.9 \text { [117.9; } \\
164.3]\end{array}$ & $\begin{array}{l}140.1 \text { [116.4; } \\
175.9]\end{array}$ & $128.1[114.4 ; 148]$ & 0.520 & & & \\
\hline $\begin{array}{l}\text { GM- } \\
\text { CSF }\end{array}$ & $87.5[62.9 ; 111.7]$ & $82.2[58.5 ; 99.2]$ & $97.3[61.4 ; 124]$ & 0.347 & & & \\
\hline IFNy & $\begin{array}{l}384.3[360.1 ; \\
425.9]\end{array}$ & $\begin{array}{l}437.5 \text { [335.5; } \\
485.7]\end{array}$ & $359.2[329.5 ; 430.4]$ & 0.433 & & & \\
\hline MCP1 & $67.2[44.2 ; 82.2]$ & $67.4[35.9 ; 96.9]$ & $74.1[56.2 ; 115.7]$ & 0.375 & & & \\
\hline MIP1 $\alpha$ & $8.2[7.6 ; 11]$ & $7.9[6.7 ; 9.4]$ & $10[7.5 ; 16.8]$ & 0.061 & & & \\
\hline MIP1 $\beta$ & $187[122 ; 253.4]$ & $\begin{array}{l}\text { 155.5 [109.7; } \\
214.3]\end{array}$ & $239[173.3 ; 400.2]$ & 0.012 & 0.561 & 0.015 & 0.111 \\
\hline TNF- $\alpha$ & $85.1[75.4 ; 100.8]$ & $88.8[71.3 ; 106.3]$ & 86.7 [72.3; 109.7] & 0.913 & & & \\
\hline $\begin{array}{l}\text { FGF } \\
\text { basic }\end{array}$ & $105.5[89.9 ; 184.4]$ & $102.7[82.5 ; 201.7]$ & $133.9[90.5 ; 210.9]$ & 0.359 & & & \\
\hline $\begin{array}{l}\text { PDGF- } \\
\mathrm{bb}\end{array}$ & $\begin{array}{l}3975.5 \text { [2970.7; } \\
5904.6]\end{array}$ & $\begin{array}{l}3339.5 \text { [479.5 ; } \\
7787.8]\end{array}$ & $\begin{array}{l}4797.8 \text { [2191.1; } \\
6099.4]\end{array}$ & 0.678 & & & \\
\hline VEGF & $295.5[128 ; 393.3]$ & $255.5[39.7 ; 419]$ & $272.1[168.1 ; 466.6]$ & 0.639 & & & \\
\hline
\end{tabular}

Table 2. Serum concentration of cytokines, chemokines and growth factors. Results are expressed as median and range [min; max] and compared between groups using the non parametric Kruskall Wallis test followed by Mann Whitney U test for pairwise comparisons.

\section{Figures}


A

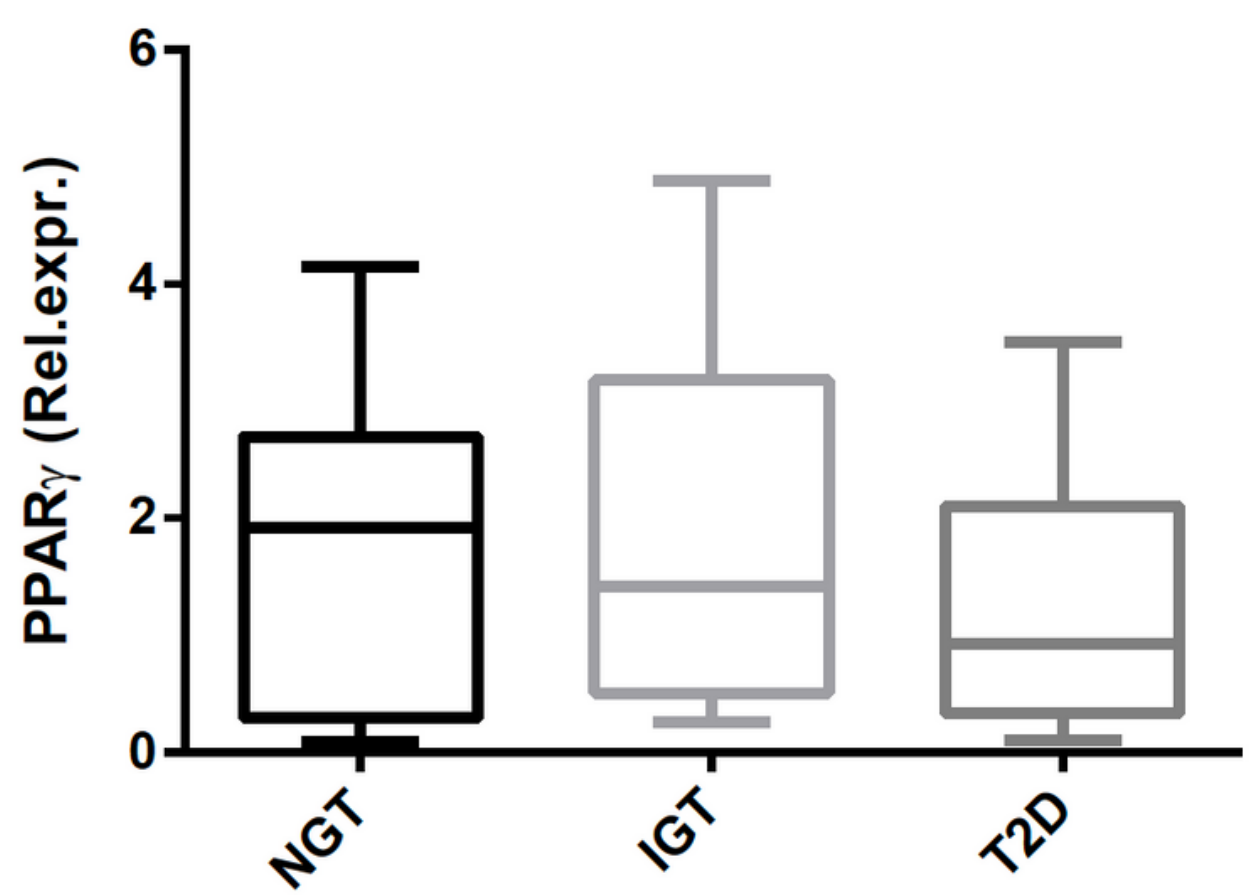

B

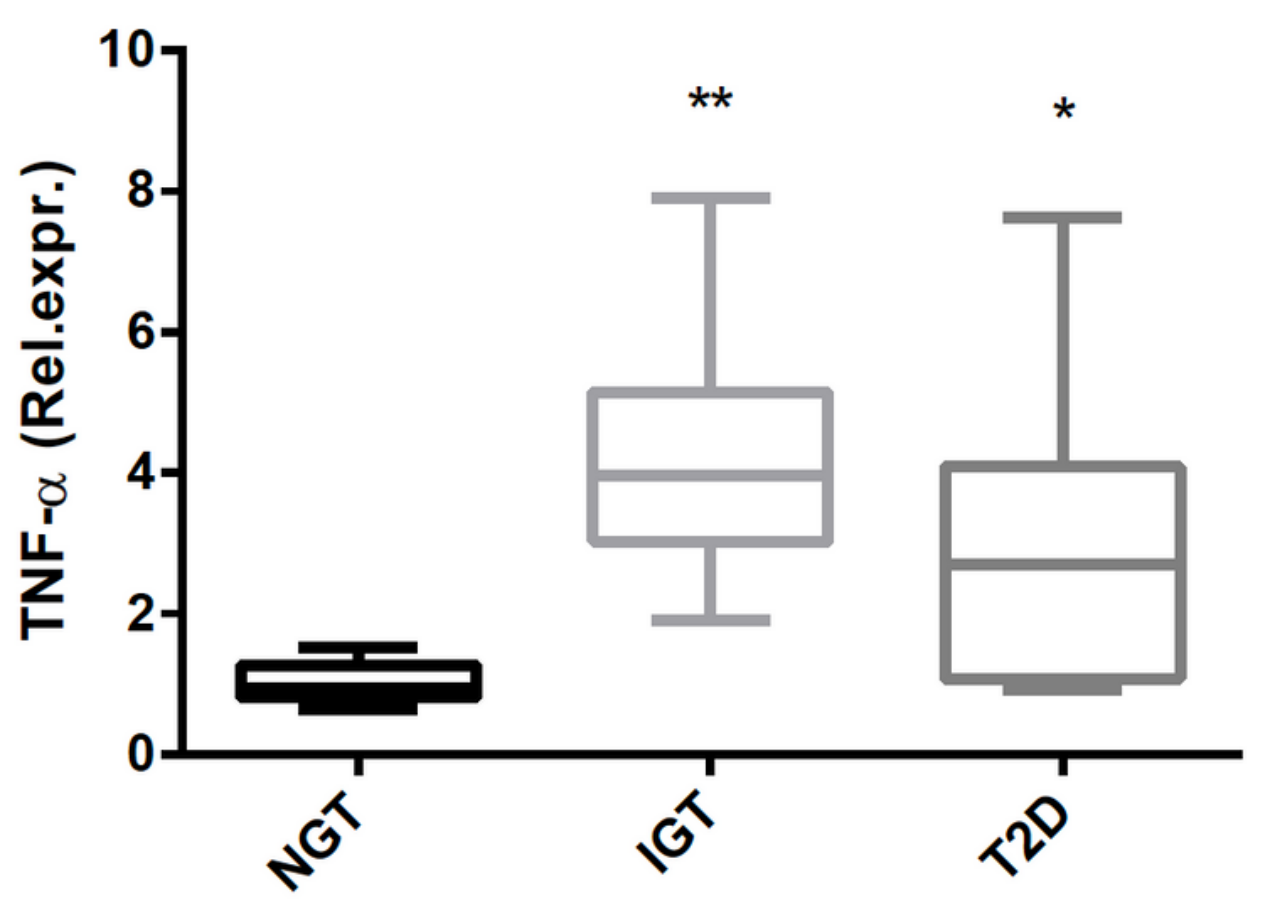

Figure 1

SAT inflammation. SAT biopsies were obtained from NGT, IGT and T2D subjects ( $\mathrm{N} \geq 6 /$ group). mRNA levels were determined by qPCR analysis of total RNA isolated from SAT biopsies. The average expression value of PPARG (A) and TNFA (B) in NGT individuals was used as reference sample and PPIA as housekeeping gene. ${ }^{*}$ Denote statistical differences vs NGT $\left({ }^{*} p<0.05 ;{ }^{* \star} p<0.01\right)$. 

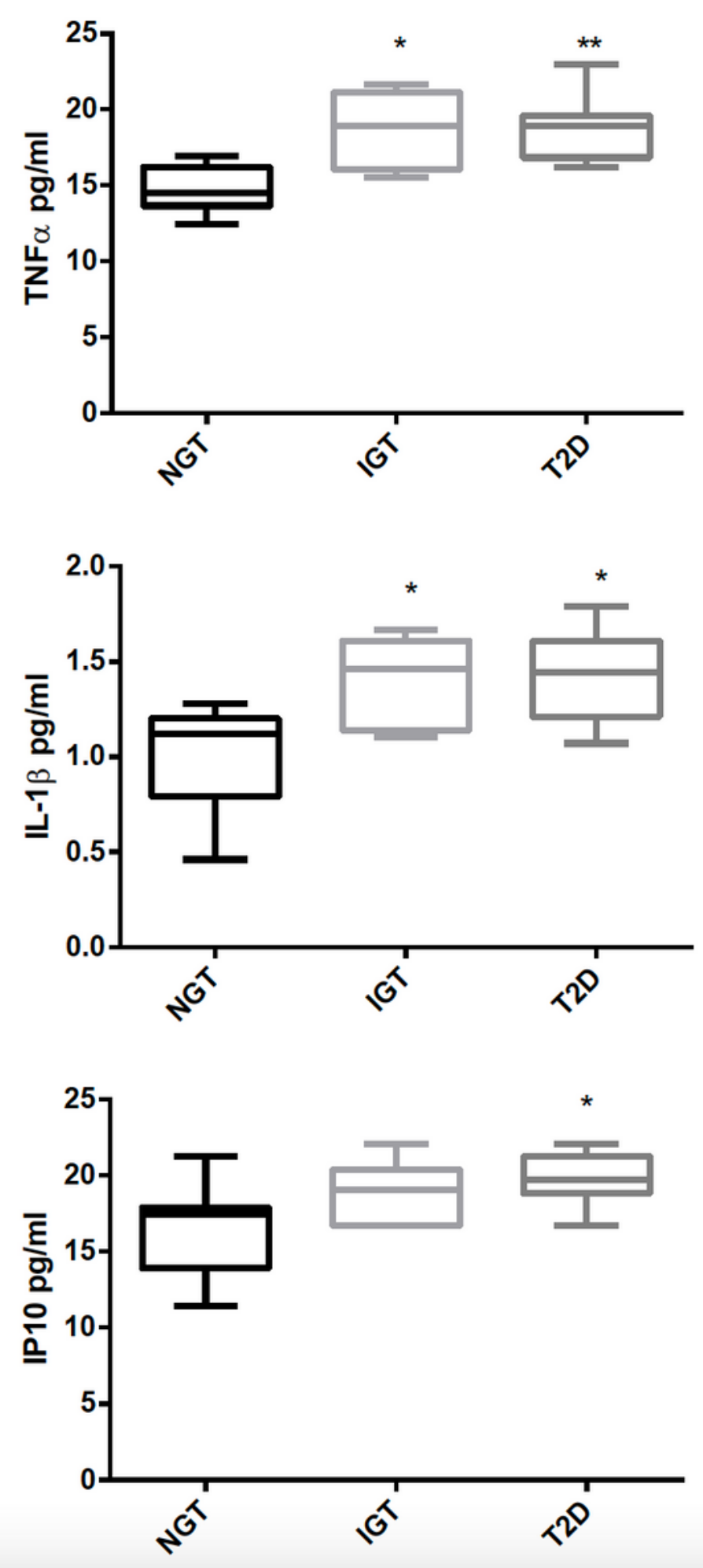

Figure 2

Adipocyte-secreted cytokines and chemokines. Adipocytes from NGT, IGT and T2D subjects ( $N \geq 10$ /group) were serum starved for $8 \mathrm{~h}$. Supernatants were collected and tested by using the Bio-Plex multiplex cytokine assay kit. *Denote statistical differences vs NGT $\left({ }^{\star} p<0.05 ;{ }^{* \star} p<0.01\right)$. 
A

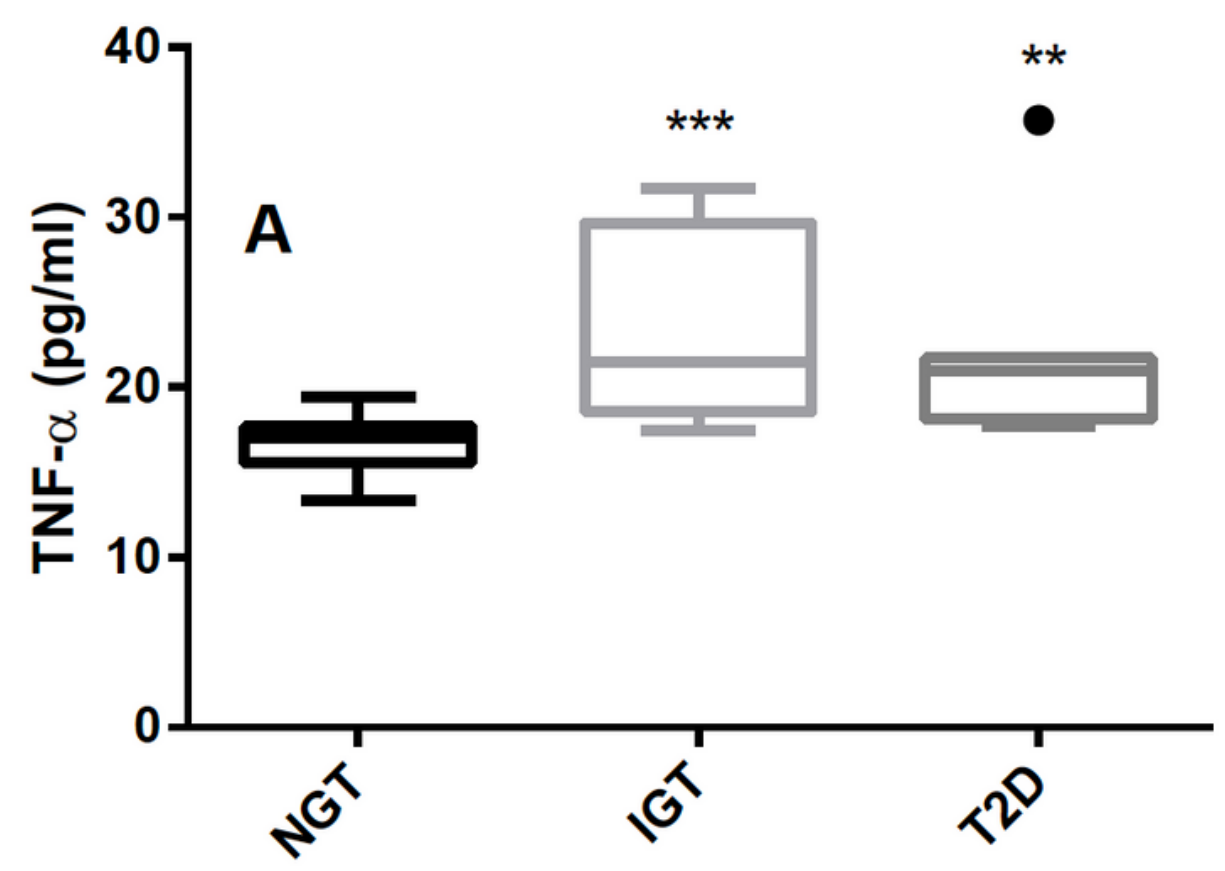

B

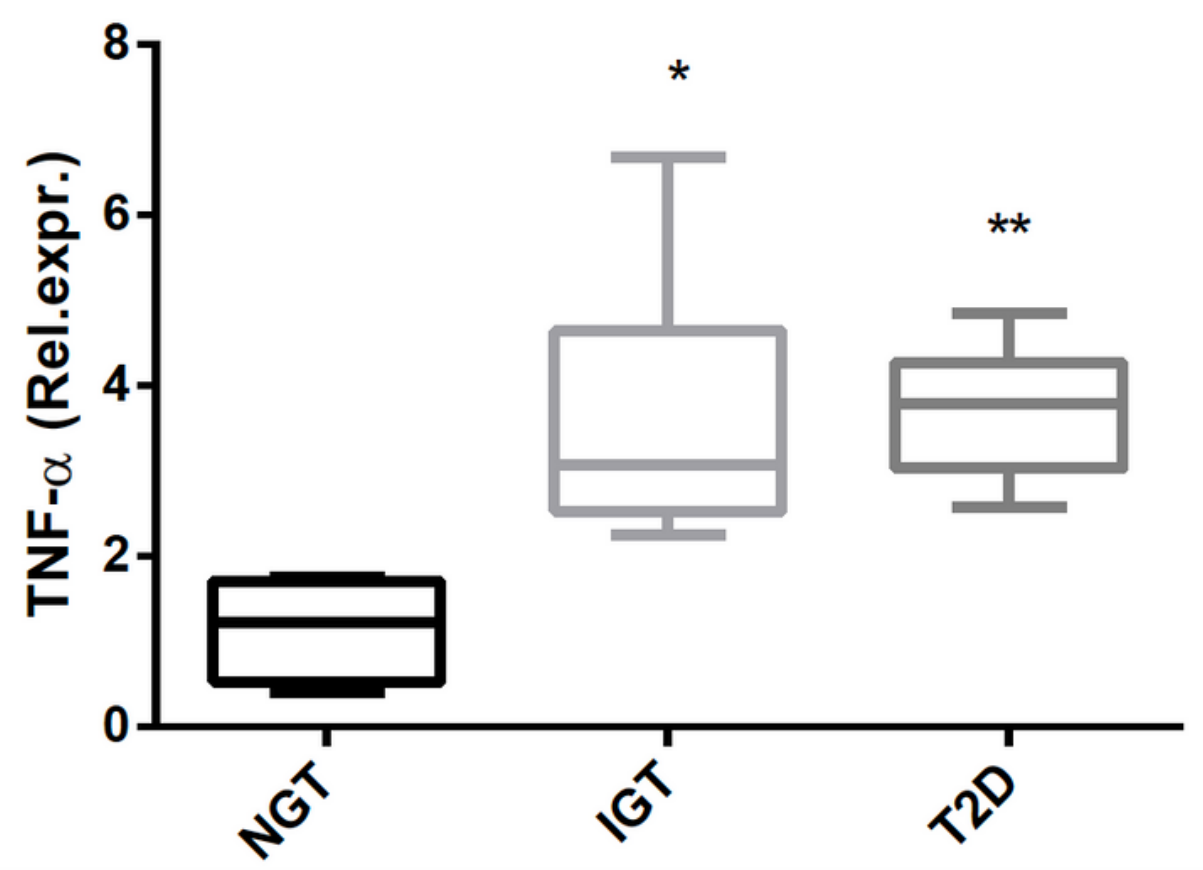

Figure 3

MSC inflammation. SAT-derived MSCs from NGT, IGT and T2D subjects ( $N \geq 4 /$ group) were analyzed for TNFA secretion (A) by using the Bio-Plex multiplex cytokine assay kit and mRNA expression (B) by qPCR analysis. The average expression value of NGT individuals was used as reference sample, and PPIA as housekeeping gene. ${ }^{*}$ Denote statistical differences vs NGT $\left({ }^{\star} p<0.05 ;{ }^{* *} p<0.01\right)$. 
A

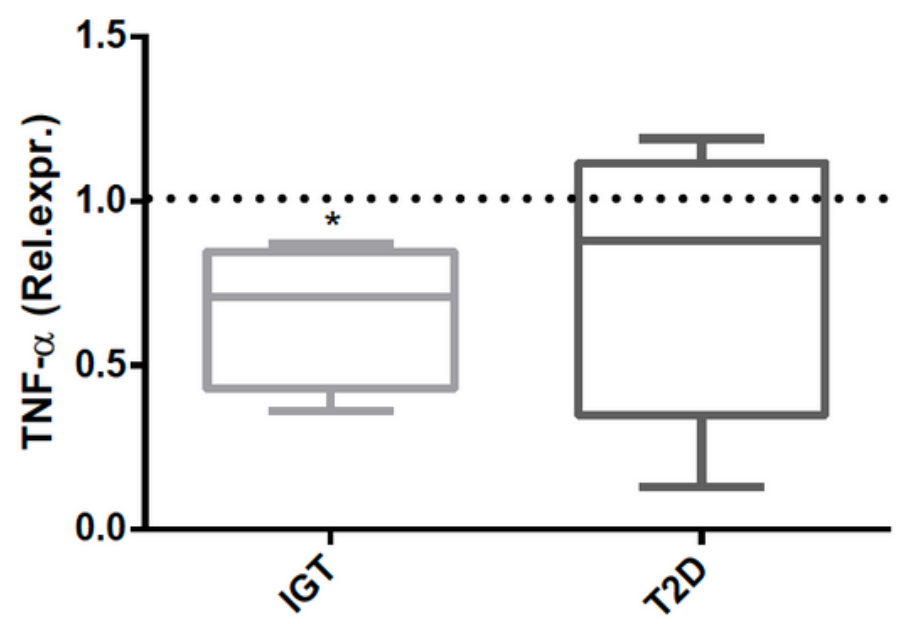

B

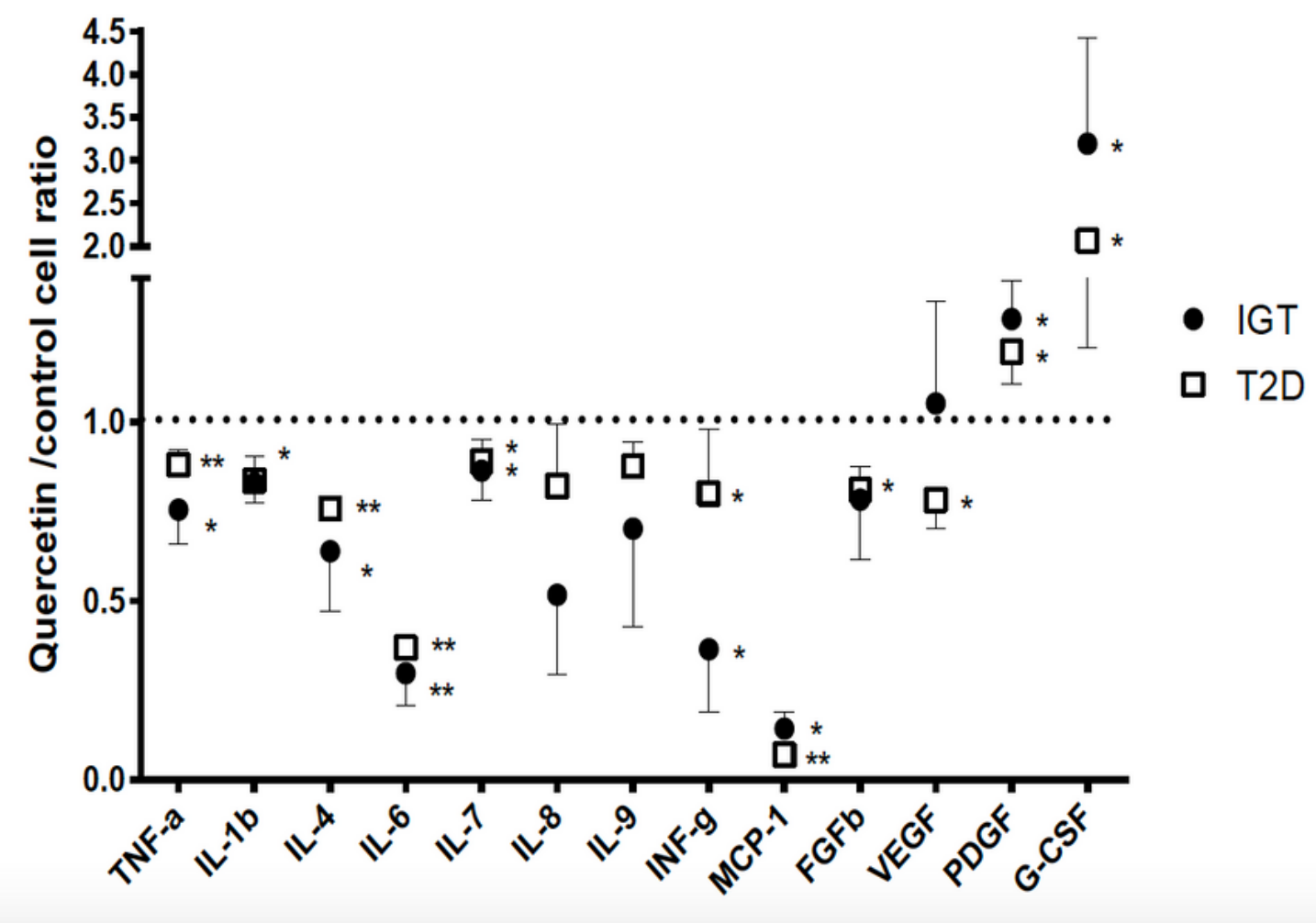

Figure 4

Quercetin effect on MSC inflammation. SAT-derived MSCs from IGT and T2D subjects ( $\geq 4$ /group) were treated with $1 \mu \mathrm{M}$ quercetin or with vehicle (DMSO), as control, for 72 hours. A) The abundance of TNF-a mRNA was determined by qRT-PCR analysis of total RNA isolated from MSCs. The average expression value of control cells (dotted line) was used as reference sample, and PPIA was used as housekeeping gene. B) Supernatants were collected and tested by using the Bio-Plex multiplex cytokine 
assay kit. Data are represented as ratio over control. *Denote statistical differences vs control cells $\left({ }^{*} p<0.05 ;{ }^{* *} p<0.01\right)$.

\section{Supplementary Files}

This is a list of supplementary files associated with this preprint. Click to download.

- SUPPLEMENTARYMATERIALS.pdf 